\title{
Estrutura da comunidade de plantas lenhosas em fragmentos de cerrado: relação com o tamanho do fragmento e seu nível de perturbação
}

\author{
ADENILZA BORGES DO CARMO ${ }^{1}$, HERALDO L. VASCONCELOS ${ }^{2} \mathrm{e}$ \\ GLEIN MONTEIRO DE ARAÚJO2,3
}

(recebido: 23 de abril de 2010; aceito: 02 de dezembro de 2010)

\begin{abstract}
Structure of the woody plant community in fragments of cerrado: relationship with fragment size and its level of disturbance). In this study we analyzed the structure of the woody plant community in 11 cerrado (savanna) fragments located in the Triângulo Mineiro region, Brazil. We evaluated whether vegetation density, diversity and/or composition were related to fragment area or its level of disturbance (cover of exotic grasses and the historical occurrence of cattle and fire). In each fragment ( 7 to 509 ha in size) 50 plots of $10 \times 10 \mathrm{~m}$ were established in order to survey all trees with a circumference at breast height $(\mathrm{CBH}) \geq 15 \mathrm{~cm}$. Tree saplings and shrubs $(\mathrm{CBH}<15 \mathrm{~cm})$ were sampled in sub-plots of $5 \times 10 \mathrm{~m}$. Larger fragments had more individuals (of saplings and shrubs), more species (of trees, saplings and shrubs), and more diversity (of trees) than the smaller fragments. Fragment area also explained part of the variation in the species composition of saplings and shrubs. With the exception of a negative relationship between the cover of exotic grasses and the density of saplings and shrubs, we did not find any correlation between the level of disturbance and the structure of the vegetation. However, it was not possible to disentangle size effects from the effects of disturbance as the smaller fragments also presented a higher degree of disturbance, particularly a greater cover of exotic grasses. These results indicate that smaller cerrado fragments are not only more vulnerable to effects resulting from habitat fragmentation, but also to the effects of invasive grasses and disturbance by fire and cattle.
\end{abstract}

Key words - alien grasses, fire disturbance, habitat fragmentation, Neotropical savannas, plant diversity

RESUMO - (Estrutura da comunidade de plantas lenhosas em fragmentos de cerrado: relação com o tamanho do fragmento e seu nível de perturbação). Neste estudo analisamos a estrutura da comunidade de plantas lenhosas em 11 fragmentos de cerrado (sensu stricto) situados no Triângulo Mineiro. Avaliamos se a densidade, diversidade e/ou composição da vegetação estavam relacionadas à área do fragmento ou ao seu grau de perturbação (medida pela cobertura de gramíneas exóticas e pela ocorrência histórica de gado e fogo). Em cada fragmento (com tamanho entre 7 a 509 ha) foram instaladas 50 parcelas de $10 \times 10 \mathrm{~m}$ para contagem e identificação das árvores com circunferência a altura do peito $(\mathrm{CAP}) \geq 15 \mathrm{~cm}$. Arbustos e arvoretas $(\mathrm{CAP}<15 \mathrm{~cm})$ foram amostrados em sub-parcelas de $5 \times 10 \mathrm{~m}$. Fragmentos maiores apresentaram maior número de indivíduos (de arbustos e arvoretas), de espécies (de árvores, arbustos e arvoretas), e maior diversidade (de árvores) do que os fragmentos menores. O tamanho do fragmento também explicou parte da variação na composição de espécies de arbustos e de arvoretas. Com exceção de uma relação negativa entre a cobertura de gramíneas e a densidade de arbustos e arvoretas, não encontramos qualquer correlação entre o grau de perturbação e a estrutura da vegetação. Entretanto, não foi possível separar os efeitos de área dos efeitos da perturbação, já que fragmentos menores tenderam a apresentar maior grau de perturbação, particularmente uma maior cobertura de gramíneas exóticas. Isto indica que fragmentos menores não apenas estão mais sujeitos aos efeitos decorrentes da fragmentação dos hábitats, mas também aos efeitos da ação do fogo, do gado e da invasão por gramíneas exóticas.

Palavras-chave - diversidade florística, fragmentação de habitats, gramíneas invasoras, perturbação por fogo, savanas Neotropicais

\section{Introdução}

O Cerrado ocupava cerca de $25 \%$ do território brasileiro, nos chapadões do planalto central (Ribeiro \& Walter 2008) em solos com boas condições para a

1. Parte da dissertação de mestrado da primeira autora, Programa de Pós Graduação em Ecologia e Conservação de Recursos Naturais, Universidade Federal de Uberlândia, Instituto de Biologia, Uberlândia, MG, Brasil.

2. Universidade Federal de Uberlândia, Instituto de Biologia, Caixa Postal 593, 38400-902 Uberlândia, MG, Brasil.

3. Autor para correspondência: glein@ufu.br agricultura e pecuária (Caruso 1997). Nos últimos 35 anos foi um dos ecossistemas com maior interferência antrópica (Aquino \& Miranda 2008), sendo hoje a região de maior produção de grãos e carne, em grande parte destinada à exportação (Tavares 2005). Em consequência disso, a região mantém atualmente $20 \%$ a $35 \%$ de sua vegetação natural e apenas $2,09 \%$ encontram-se oficialmente em unidades de conservação (Mittermeier et al. 1999, Klink \& Machado 2005). A expansão da fronteira agrícola no Cerrado - cuja flora é considerada uma das mais ricas e com maior grau de endemismo entre as savanas tropicais do mundo (Myers et al. 2000, 
Ribeiro \& Walter 2008) - além de causar perda de habitat resultou na fragmentação da vegetação remanescente (Aquino \& Miranda 2008, Carvalho et al. 2009).

A perda e a fragmentação dos habitats naturais constituem hoje uma das maiores ameaças à biodiversidade (Fahrig 2003). Estudos na Amazônia mostram que a fragmentação da floresta causa efeitos diversos e severos sobre a comunidade vegetal (revisão em Laurance et al. 2002, Laurance \& Vasconcelos 2009). Fragmentos menores têm maior taxa de mortalidade de árvores do que fragmentos maiores ou áreas não fragmentadas (Laurance et al. 2002, Laurance \& Vasconcelos 2009). A formação de clareiras resultante desta maior mortandade nos fragmentos, especialmente próximo à borda dos mesmos, favorece o recrutamento de espécies de plantas pioneiras em detrimento das espécies típicas da floresta não perturbada (Laurance et al. 2006). Consequentemente há uma alteração na composição florística e redução na diversidade de espécies (Laurance et al. 2006).

Embora os efeitos da fragmentação sobre a flora de florestas tropicais estejam relativamente bem documentados (e.g., Laurance 1999, Tabarelli et al. 1999, Pimm \& Raven 2000, Laurance et al. 2002, Laurance \& Vasconcelos 2009), há ainda muitas incertezas sobre como e se estes efeitos atuam sobre as savanas tropicais. Por exemplo, embora inúmeros trabalhos tenham analisado os efeitos de borda em fragmentos de floresta tropical (Laurance et al. 2002), fomos capazes de encontrar apenas um artigo sobre este assunto para o Cerrado (Lima-Ribeiro 2008). Ainda assim, o referido trabalho trata dos efeitos de borda sobre a vegetação de cerradão (Lima-Ribeiro 2008), que efetivamente é uma fisionomia florestal do bioma Cerrado (Ribeiro \& Walter 2008).

Um dos problemas em se estudar os efeitos da fragmentação do habitat em paisagens já muito alteradas como as do bioma Cerrado é que boa parte dos remanescentes vegetais existentes já sofreram algum grau de interferência humana. Assim, eventuais mudanças na biota dos fragmentos podem ser decorrentes tanto da fragmentação em si como de outras formas de distúrbio, como o corte seletivo de madeira, o fogo, o pastejo pelo gado e a invasão de espécies exóticas. De fato, as gramíneas invasoras de origem africana são hoje encontradas em praticamente todos os fragmentos de cerrado (Pivello et al. 1999). A invasão destas gramíneas parece ser facilitada pela presença de rodovias, de áreas urbanas ou degradadas (Hoffmann et al. 2004, Martins et al. 2004, Durigan et al. 2007) e pelo gado que dissemina as suas sementes (Bruun \& Fritzboger 2002). Além disto, algumas gramíneas invasoras, como Brachiaria decumbens, têm ação alelopática o que aumenta o seu poder competitivo com as espécies de gramíneas nativas (Barbosa et al. 2008). As gramíneas invasoras, por sua vez, favorecem a ocorrência de fogo (Moreira 2000) e o pastejo de gado bovino (Vieira et al. 2006), criando assim feedbacks positivos entre as diferentes formas de distúrbio.

Neste estudo analisamos a estrutura da vegetação lenhosa em 11 fragmentos de cerrado (sensu stricto) de diferentes tamanhos e com diferentes graus de perturbação antrópica (perturbação esta medida pela cobertura de gramíneas exóticas, pela ocorrência de gado e pela ocorrência de incêndios). O objetivo foi determinar se a densidade, diversidade e/ou composição da vegetação variava em função da área do fragmento e de seu grau de perturbação. Nossa hipótese é que a perda de área e o aumento da perturbação afetam a estrutura da vegetação de cerrado, acarretando uma perda de espécies e alteração na composição da comunidade nos fragmentos de menor tamanho ou com maior grau de perturbação.

\section{Material e Métodos}

O estudo foi realizado em onze fragmentos de cerrado (sensu stricto), segundo Coutinho (1978), sendo 10 deles situados no município de Uberlândia e um em Uberaba, Minas Gerais. Os fragmentos possuem áreas que variam de 7 a 509 ha (tabela 1). A maioria destes remanescentes é reserva legal de propriedades particulares e em sua periferia encontram-se áreas agrícolas ou pastagens. O clima da região é do tipo Aw (Köppen 1936), com verões quentes e úmidos, e invernos frios e secos. A precipitação pluviométrica anual é de cerca de $1500 \mathrm{~mm}$ e a temperatura média de $22{ }^{\circ} \mathrm{C}$ (Silva et al. 2008). Todos os fragmentos estudados ocorriam sobre Latossolos Vermelho-Escuros, que são solos moderadamente a fortemente ácidos (Embrapa 1982).

Para a amostragem da vegetação foram instaladas aleatoriamente em cada fragmento, a uma distância de cerca de $5 \mathrm{~m}$ da borda, 50 parcelas contíguas de $10 \times 10 \mathrm{~m}$ (totalizando assim uma área amostral de 0,5 ha por fragmento). Em cada parcela de $10 \times 10 \mathrm{~m}$ foram identificados e contados todas as árvores com circunferência a altura do peito (CAP) $\geq 15 \mathrm{~cm}$ a 1,3 $\mathrm{m}$ do solo. Para amostragem dos arbustos e arvoretas (indivíduos com $\mathrm{CAP}<15 \mathrm{~cm}$ e altura acima de um metro) foi estabelecida uma sub-parcela de $5 \times 10 \mathrm{~m}$ dentro de cada parcela, totalizando uma área de amostragem 0,25 ha. $\mathrm{O}$ procedimento de sub-divisão da parcela maior foi utilizado para facilitar a amostragem da vegetação.

Adotou-se a técnica de Avaliação Rápida Participativa (Rapid Participatory Appraisal, Chambers 1987, 1992), para estimar a cobertura de gramíneas invasoras nos fragmentos 
Tabela 1. Características dos fragmentos de cerrado (sensu stricto) estudados nos Municípios de Uberlândia e Uberaba, MG.

Table 1. Characteristics of the studied cerrado (savanna) fragments in the municipality of Uberlândia and Uberaba, MG.

\begin{tabular}{|c|c|c|c|c|c|}
\hline $\begin{array}{l}\text { Área } \\
\text { (ha) }\end{array}$ & $\begin{array}{c}\text { Cobertura de } \\
\text { gramíneas exóticas* }\end{array}$ & $\begin{array}{l}\text { Ocorrência de } \\
\text { gado** }\end{array}$ & $\begin{array}{l}\text { Ocorrência de } \\
\text { fogo } * *\end{array}$ & $\begin{array}{l}\text { Latitude } \\
\text { (S) }\end{array}$ & $\begin{array}{l}\text { Longitude } \\
\text { (W) }\end{array}$ \\
\hline 7 & 4 & 2 & 3 & $19^{\circ} 01^{\prime} 25^{\prime \prime}$ & $48^{\circ} 04^{\prime} 23^{\prime \prime}$ \\
\hline 10 & 4 & 4 & 3 & $19^{\circ} 02^{\prime} 06^{\prime \prime}$ & $48^{\circ} 32^{\prime} 05^{\prime \prime}$ \\
\hline 40 & 1 & 0 & 0 & $19^{\circ} 11^{\prime} 01^{\prime \prime}$ & $48^{\circ} 10^{\prime} 28^{\prime \prime}$ \\
\hline 50 & 2 & 1 & 2 & $19^{\circ} 02^{\prime} 55^{\prime \prime}$ & $48^{\circ} 02^{\prime} 38^{\prime \prime}$ \\
\hline 60 & 1 & 0 & 0 & $19^{\circ} 05^{\prime} 44^{\prime \prime}$ & $48^{\circ} 21^{\prime} 03^{\prime \prime}$ \\
\hline 96 & 1 & 0 & 0 & $19^{\circ} 05^{\prime} 39^{\prime \prime}$ & $48^{\circ} 08^{\prime} 46^{\prime \prime}$ \\
\hline 151 & 1 & 2 & 0 & $19^{\circ} 00^{\prime} 35^{\prime \prime}$ & $48^{\circ} 35^{\prime} 05^{\prime \prime}$ \\
\hline 170 & 0 & 0 & 0 & $19^{\circ} 02^{\prime} 25^{\prime \prime}$ & $48^{\circ} 05^{\prime} 46^{\prime \prime}$ \\
\hline 176 & 2 & 0 & 1 & $19^{\circ} 08^{\prime} 46^{\prime \prime}$ & $48^{\circ} 22^{\prime} 41^{\prime \prime}$ \\
\hline 441 & 1 & 1 & 2 & $19^{\circ} 00^{\prime} 06^{\prime \prime}$ & $48^{\circ} 18^{\prime} 38^{\prime \prime}$ \\
\hline 509 & 0 & 1 & 0 & $19^{\circ} 10^{\prime} 23^{\prime \prime}$ & $48^{\circ} 23^{\prime} 58^{\prime \prime}$ \\
\hline
\end{tabular}

* 0 = ausência; $1=$ de 1 a $25 \% ; 2=$ de 26 a $50 \% ; 3=$ de 51 a $75 \%$ e $4=$ mais de $75 \%$ da área invadida. ** $0=$ sem registro de ocorrência há pelo menos 20 anos; $1=$ ocorrência entre 10 a 20 anos; 2 = ocorrência entre 5 a 10 anos; $3=$ uma única ocorrência a menos de 5 anos; $4=$ mais de uma ocorrência a menos de 5 anos.

$* 0=$ absence; $1=$ from 1 to $25 \% ; 2=$ from 26 to $50 \% ; 3=$ from 51 to $75 \% ; 4=$ more than $75 \%$ of the area invaded. $* * 0=$ withouth register of occurrence for at least 20 years; 1 = occurrence from 10 to 20 years; $2=$ occurrence from 5 to 10 years; $3=$ one single occcurrence in less 5 years; $4=$ more than one occurrence in less 5 years.

(que nas áreas consistia em sua maioria de Brachiaria decumbens Stapf e eventualmente Melinis minutiflora P. Beauv.) e, também, para determinar o tempo desde a última ocorrência de fogo e da utilização da área como pastagem para o gado. A cobertura de capim exótico foi estimada visualmente (e sempre pela mesma pessoa) dentro das parcelas amostrais, utilizando-se as seguintes categorias: $0=$ ausência, $1=$ entre 1 a $25 \%, 2=$ entre 26 a $50 \%, 3=$ entre 51 a $75 \%$ e $4=$ mais de $75 \%$ da área invadida. Para determinar o tempo desde a última ocorrência de fogo e do uso da área como pastagem, foram feitas entrevistas com os proprietários das áreas onde os fragmentos estavam situados. Tanto para fogo quanto para gado foram usadas as seguintes categorias: $0=$ sem registro de ocorrência há pelo menos 20 anos, $1=$ ocorrência entre 10 a 20 anos, $2=$ ocorrência entre 5 a 10 anos, 3 = uma única ocorrência a menos de 5 anos e $4=$ mais de uma ocorrência a menos de 5 anos. Análises de correlação foram feitas para avaliar a possível influência do tamanho do fragmento, da ocorrência de fogo, da ocorrência de gado e da cobertura de gramíneas exóticas sobre a abundância de indivíduos, a densidade de espécies e diversidade de espécies. Para tanto, utilizou-se a análise de correlação por ranks (Correlação de Spearman; Siegel 1956), uma vez que os dados sobre a ocorrência de fogo, gado e cobertura de gramíneas exóticas foram medidos em escala ordinal. Foram feitas análises em separado para árvores (indivíduos com CAP $\geq 15 \mathrm{~cm}$ ) e para arbustos e arvoretas (indivíduos com $<15 \mathrm{~cm}$ de CAP).

Como medida da densidade (ou riqueza) de espécies em cada fragmento usou-se o número total de espécies registradas nas 50 parcelas amostradas. Como medida de diversidade calculou-se o índice Alpha de Fisher (Fisher et al. 1943). Esse índice tem algumas vantagens sobre outros índices de diversidade, como os índices de Shannon e de Simpson, por ser pouco influenciado pelo tamanho da amostra ou pela abundância das espécies mais comuns (Taylor et al. 1976). $\mathrm{O}$ índice Alpha de Fisher relaciona o número de espécies $(S)$ ao número de indivíduos $(N)$ em uma comunidade através da seguinte equação: $S=\alpha \ln (1+N / \alpha)$ (Fisher et al. 1943).

Um escalonamento não-métrico multidimensional (nonmetric multidimensional scaling ou MDS) foi utilizado, para identificar as variáveis ambientais (área e grau de perturbação) correlacionadas com a composição de espécies de plantas nos fragmentos. A técnica de ordenação MDS foi escolhida em detrimento de outras técnicas de ordenação, pois o MDS é considerado por muitos autores como mais efetivo para dados ecológicos de comunidades, já que ele não tem como premissa que existe uma relação linear entre as variáveis (McCune \& Grace 2002). As ordenações foram feitas com o uso do software Systat v.10 (SPSS 2000), tendo como base os dados de abundância relativa das espécies em cada fragmento e medida de similaridade o índice Bray-Curtis. Análises da correlação entre os scores produzidos pela ordenação e as variáveis ambientais foram feitas em separado para árvores e para arbustos e arvoretas.

\section{Resultados}

Entre os fragmentos amostrados, observou-se que aqueles com um histórico mais recente de perturbação 
por fogo apresentaram também um histórico mais recente de utilização como pastagem para o gado, assim como uma maior cobertura de gramíneas exóticas (tabela 2). Não houve correlação significativa entre o tamanho do fragmento e a ocorrência de fogo nem entre o tamanho do fragmento e a ocorrência de gado. Porém, foi observada uma correlação negativa e significativa entre o tamanho do fragmento e e a cobertura de gramíneas exóticas (tabela 2).

Um total de 18.703 indivíduos distribuídos em 168 espécies foram amostrados nos 11 fragmentos (tabela 3 ). Em 11.438 indivíduos com CAP $<15 \mathrm{~cm}$ encontrou-se 164 espécies e em 7.265 indivíduos com CAP $\geq 15 \mathrm{~cm}$ registrou-se 106 espécies. O número de espécies de

Tabela 2. Coeficientes de correlação de Spearman entre a área do fragmento, a cobertura de gramíneas exóticas, a ocorrência de gado e a ocorrência de fogo.

Table 2. Spearman correlation coefficients between fragment area, cover of exotic grasses, occurrence of cattle, and occurrence of fire.

\begin{tabular}{lccc}
\hline & $\begin{array}{c}\text { Área } \\
\text { (ha) }\end{array}$ & $\begin{array}{c}\text { Cobertura de } \\
\text { gramíneas } \\
\text { exóticas }\end{array}$ & $\begin{array}{c}\text { Ocorrência } \\
\text { de gado }\end{array}$ \\
\hline Cobertura de & $-0,677^{*}$ & - & - \\
gramíneas exóticas & $-0,290$ & 0,477 & - \\
Ocorrência de gado & $-0,290$ & $0,835^{*}$ & $0,625^{*}$ \\
\hline
\end{tabular}

$* P<0,05$. árvores encontradas por fragmento variou de 43 a 63 e de 56 a 92 para arbustos e arvoretas (tabela 3 ).

Para arbustos e arvoretas houve uma correlação positiva e significativa entre o tamanho do fragmento e a densidade de indivíduos e de espécies (tabela 4). Para árvores foram encontradas correlações positivas e significativas entre o tamanho do fragmento e a densidade e diversidade de espécies (tabela 4). A cobertura de gramíneas exóticas apresentou correlação significativa apenas com a densidade de arbustos e arvoretas. Fragmentos com maior cobertura de gramíneas exóticas tenderam a apresentar menor densidade de arbustos e arvoretas (figura 1). Já em relação à ocorrência de gado ou fogo nenhuma correlação significativa foi detectada (tabela 4).

A similaridade na composição florística dos fragmentos foi relativamente baixa (Índice Bray-Curtis: arbustos e arvoretas $=0,316 \pm 0,084$; árvores $=$ $0,312 \pm 0,087)$, o que sugere que as espécies têm distribuição bastante heterogênea. Nem a cobertura de gramíneas nem a ocorrência de gado e fogo apresentaram correlações significativas com os scores produzidos pela ordenação dos fragmentos em função de suas similaridades na composição de espécies (figura 2), tanto de árvores quanto de arbustos e arvoretas (tabela 4). Já o tamanho do fragmento apresentou correlação com os scores da ordenação, porém apenas para a ordenação com dados de arbustos e arvoretas (tabela 4). A maior parte dos fragmentos de menor tamanho ( $\leq 60$ ha) estão posicionados na margem esquerda do gráfico de ordenação, enquanto os fragmentos de maior

Tabela 3. Estrutura da comunidade de plantas lenhosas em fragmentos de cerrado (sensu stricto) de diferentes tamanhos.

Table 3. Structure of the woody plant community in cerrado fragments of different sizes.

\begin{tabular}{|c|c|c|c|c|c|c|}
\hline \multirow{2}{*}{$\begin{array}{l}\text { Área do } \\
\text { fragmento } \\
\text { (ha) }\end{array}$} & \multicolumn{3}{|c|}{ Árvores } & \multicolumn{3}{|c|}{ Arvoretas e arbustos } \\
\hline & $\begin{array}{l}\text { Espécies } \\
(0,5 \text { ha })\end{array}$ & $\begin{array}{l}\text { Indivíduos } \\
\text { (0,5 ha) }\end{array}$ & $\begin{array}{c}\text { Diversidade } \\
\text { (alpha de Fisher) }\end{array}$ & $\begin{array}{l}\text { Espécies } \\
(0,25 \text { ha })\end{array}$ & $\begin{array}{l}\text { Indivíduos } \\
(0,25 \text { ha })\end{array}$ & $\begin{array}{c}\text { Diversidade } \\
\text { (alpha de Fisher) }\end{array}$ \\
\hline 7 & 43 & 614 & 10,53 & 60 & 1042 & 13,84 \\
\hline 10 & 48 & 687 & 11,74 & 56 & 350 & 18,82 \\
\hline 40 & 46 & 511 & 12,25 & 60 & 699 & 15,71 \\
\hline 50 & 49 & 522 & 13,24 & 92 & 1070 & 22,71 \\
\hline 60 & 52 & 622 & 13,5 & 69 & 930 & 17,21 \\
\hline 96 & 54 & 665 & 13,88 & 77 & 1079 & 18,97 \\
\hline 151 & 54 & 634 & 12,05 & 82 & 1082 & 20,6 \\
\hline 170 & 44 & 656 & 10,63 & 88 & 1402 & 22,07 \\
\hline 176 & 48 & 545 & 14,88 & 72 & 603 & 21,34 \\
\hline 441 & 59 & 948 & 13,93 & 89 & 1131 & 22,64 \\
\hline 509 & 63 & 861 & 15,32 & 86 & 2050 & 18,16 \\
\hline
\end{tabular}


tamanho estão situados à direita (figura 2A), indicando que fragmentos de diferentes tamanhos tendem a diferir em sua composição de espécies de arbustos e arvoretas.

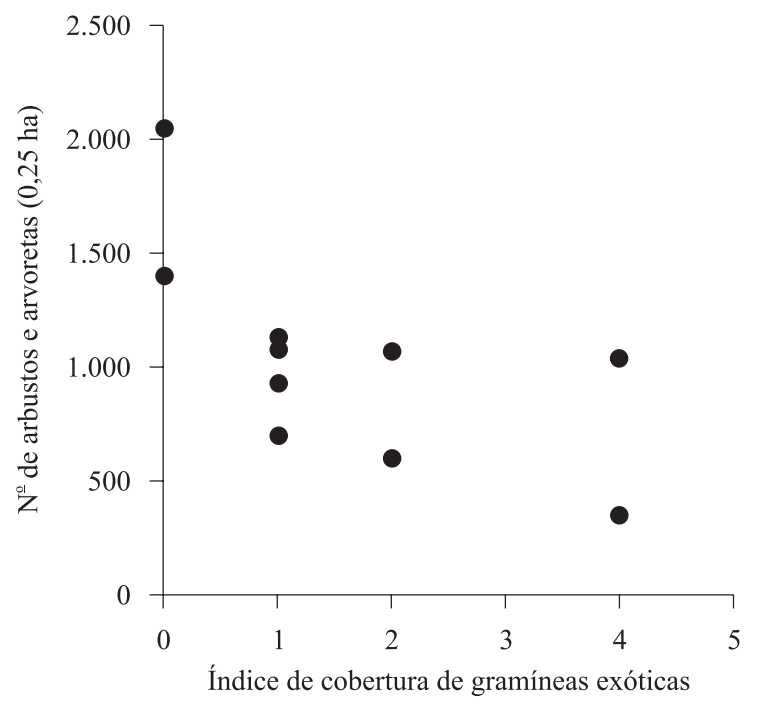

Figura 1. Relação entre a cobertura de gramíneas exóticas invasoras em fragmentos de cerrado (sensu stricto) de diferentes tamanhos e a densidade de arbustos e arvoretas.

Figure 1. Relationship between the cover of invasive alien grasses and the density of shrubs and saplings in cerrado (savanna) fragments of different sizes.

\section{Discussão}

O Cerrado é considerado um dos ecossistemas mais fragmentados nos últimos 25 anos com impacto marcante em sua biodiversidade (Ratter et al. 1996; 1997). Atualmente fragmentos de diversos tamanhos e formas distribuem-se em uma matriz de pastagens e agricultura (Carvalho et al. 2009) e em geral estão submetidos aos impactos do fogo, do gado e gramíneas exóticas (Pivello et al. 1999, Hoffmann 2002, Durigan et al. 2007). Os resultados de nosso estudo na região do Triângulo Mineiro indicam que o tamanho do fragmento é um importante preditor da estrutura da comunidade de plantas lenhosas em fragmentos de cerrado. Fragmentos maiores apresentaram maior densidade de indivíduos (de arbustos e arvoretas), maior densidade de espécies (de árvores, arbustos e arvoretas), e maior diversidade de espécies (de árvores). Além disto, o tamanho do fragmento parece explicar parte da variação na composição florística dos fragmentos, particularmente na composição de espécies de arbustos e de arvoretas.

Estudos em florestas tropicais mostram que quanto menor o fragmento maior a influência dos efeitos de borda, os quais causam fortes alterações microclimáticas, levando a extinção das espécies de interior e predomínio das espécies de borda, diminuindo, com isso, a diversidade florística (Terborgh 1992, Laurance et al. 2002). Fragmentos florestais de tamanho

Tabela 4. Relação entre a área do fragmento e seu nível de perturbação (cobertura de gramíneas exóticas, ocorrência de gado e fogo) com a densidade, riqueza, diversidade e composição de espécies (scores da ordenação MDS) de arbustos e arvoretas e de árvores. Os valores representam os coeficientes de correlação de Spearman.

Table 4. Relationship between fragment area and its level of disturbance (cover of exotic grasses, occurrence of cattle and fire) with the density, richness, diversity, and species composition (MDS ordination scores) of trees, shrubs and tree saplings. Values represent Spearman correlation coefficients.

\begin{tabular}{llccrr}
\hline Grupo de plantas & Variável & $\begin{array}{c}\text { Área } \\
\text { (ha) }\end{array}$ & Gramíneas exóticas & Gado & Fogo \\
\hline Arbustos e arvoretas & Densidade de indivíduos & $0,645^{*}$ & $-0,754^{*}$ & $-0,014$ & $-0,409$ \\
& Densidade de espécies & $0,638^{*}$ & $-0,484$ & $-0,119$ & $-0,180$ \\
& Diversidade & 0,482 & $-0,115$ & $-0,058$ & 0,100 \\
& MDS eixo 1 & $0,700^{*}$ & $-0,432$ & 0,241 & $-0,184$ \\
& MDS eixo 2 & $-0,055$ & $-0,144$ & $-0,381$ & $-0,473$ \\
Árvores & Densidade de indivíduos & 0,500 & $-0,351$ & 0,299 & 0,015 \\
& Densidade de espécies & $0,712^{*}$ & $-0,294$ & $-0,177$ & $-0,168$ \\
& Diversidade & $0,709^{*}$ & $-0,322$ & $-0,362$ & $-0,269$ \\
& MDS eixo 1 & 0,409 & $-0,178$ & 0,425 & 0,001 \\
& MDS eixo 2 & $-0,236$ & 0,480 & 0,425 & 0,219 \\
\hline
\end{tabular}

$* P<0,05$. 



Figura 2. Escalonamento multidimensional não-métrico (MDS) de 11 fragmentos de cerrado (sensu stricto) em função da composição de espécies (abundância relativa de cada espécie) de arbustos e arvoretas (A) e árvores (B). Os números ao lado dos símbolos representam a área (em hectares) de cada fragmento.

Figure 2. Non-metric multdimensional scaling (MDS) of 11 cerrado fragments based on the species composition (relative abundance of each species) of shrubs and saplings (A) and trees (B). Numbers beside the symbols represent the area (in hectares) of each fragment.

reduzido também estão mais sujeitos aos efeitos de amostragem (Laurance \& Vasconcelos 2009), ou seja, de haver uma maior probabilidade de ocorrência de espécies com padrão agregado de distribuição em fragmentos maiores. É bem provável que fragmentos de cerrado também estejam sujeitos aos efeitos de amostragem, especialmente considerando que neste tipo de vegetação o padrão de distribuição das espécies é agrupado (Meirelles \& Barreto Luiz 1995, Hay et al. 2000) e há um grande número de espécies raras (Ratter et al. 2003). Entretanto, é improvável que as variações aqui observadas na estrutura da vegetação arbóreo-arbustiva em fragmentos de cerrado sejam resultantes dos efeitos de borda. Isto porque nossas parcelas amostrais foram instaladas sempre a uma mesma distância da borda (a cerca de $5 \mathrm{~m}$ da borda) de cada um dos fragmentos, independente do tamanho do fragmento. Além disto, estudos em fragmentos de cerradão indicam um aumento na densidade arbórea próximo à borda (Lima-Ribeiro 2008). Este resultado é oposto ao apresentado aqui já que observamos que fragmentos menores (e, portanto, mais suscetíveis aos efeitos de borda) apresentam menor densidade de arbustos e arvoretas que os fragmentos de maior tamanho.

$\mathrm{Na}$ maioria dos casos não foi observada uma correlação significativa entre o grau de perturbação dos fragmentos e a estrutura da vegetação arbóreo-arbustiva. Entretanto, não podemos descartar a possibilidade de que as diferenças observadas na estrutura da vegetação entre fragmentos de diferentes tamanhos sejam na verdade resultado da ação dos agentes de perturbação. Isto porque os fragmentos menores tenderam a apresentar maior grau de perturbação, particularmente uma maior cobertura de gramíneas exóticas invasoras. Esta relação pode ajudar a explicar porque nos fragmentos menores foi encontrada uma menor densidade e riqueza de espécies de arbustos e arvoretas, já que as gramíneas exóticas invasoras podem inibir competitivamente o estabelecimento de plantas lenhosas (Hoffmann et al. 2004). Além disto, as gramíneas exóticas invasoras em geral acumulam mais biomassa combustível que as gramíneas nativas e assim as áreas invadidas por gramíneas exóticas tendem a ter maior chance de incêndios e incêndios de maior intensidade (Pivello et al. 1999, Hoffmann et al. 2004). Assim como as gramíneas invasoras, o fogo tem efeito negativo sobre a vegetação arbórea de cerrado, pois causa aumento na taxa de mortalidade, especialmente entre os indivíduos de menor porte (Medeiros \& Miranda 2005).

Em conclusão, nossos resultados dão apoio à hipótese de que a perda de área e o aumento da perturbação afetam a estrutura da vegetação de cerrado, levando a um empobrecimento desta vegetação e acarretando mudanças na sua composição. Embora diversos estudos 
mostrem que a fragmentação dos habitats, assim como as perturbações causadas pelo pastoreio, fogo e espécies invasoras têm efeitos deletérios sobre as comunidades vegetais (Tabarelli et al. 1999, Bruun \& Fritzboger 2002, Laurance et al. 2002, Hoffmann et al. 2004), encontramos aqui evidências de que estes fatores agem de forma conjunta nos remanescentes de vegetação de cerrado. Isto sugere que fragmentos menores não apenas estão mais sujeitos aos diversos efeitos decorrentes da fragmentação dos habitats (i.e., perda de área, efeito amostral, maior susceptibilidade aos efeitos de borda, maior grau de isolamento), mas também estão mais sujeitos à ação do fogo, gado e invasão por gramíneas exóticas. Medidas simples como a construção e manutenção de cercas e de aceiros no entorno destes fragmentos poderiam ajudar a mitigar estes efeitos e, assim, aumentar o seu valor de conservação.

Agradecimentos - A Mariana Rezende e Bruno Pereira pela ajuda no trabalho de campo.

\section{Referências bibliográficas}

AQUINO, F.G. \& MIRANDA, H.B.M. 2008. Consequências ambientais da fragmentação de habitats no Cerrado. In Cerrado: ecologia e flora. (S.M. Sano \& S.P. Almeida, eds.). Embrapa-CPAC, Planaltina, p.385-398.

BARBOSA, E.G., PIVELLO, V.R. \& MEIRELLES, S.T. 2008. Allelopathic evidence in Brachiaria decumbens and its potential to invade the Brazilian cerrados. Brazilian Archives of Biology and Technology 51:825831.

BRUUN, H.H. \& FRITZBOGER, B. 2002. The past impact of livestock husbandry on dispersal of plant seeds in the landscape of Denmark. Ambio 31:425-431.

CARUSO, R. 1997. The Brazilian cerrado: development, conservation and sustainability. Campinas, Fundação Cargill.

CARVALHO, F.M.V., MARCO JUNIOR, P. \& FERREIRA, L.G. 2009. The Cerrado into-pieces: Habitat fragmentation as a function of landscape use in the savannas of Brazil. Biological Conservation 142:13921403.

CHAMBERS, R. 1987. Rural development: putting the last first. Longman Scientific and Technical, New York.

CHAMBERS, R. 1992. Rural appraisal: rapid, relaxed and participatory (IDS discussion Papers 311) Institute of Development Studies, London.

COUTINHO, L.M. 1978. O conceito de cerrado. Revista Brasileira de Botânica 1:17-23.

DURIGAN, G., SIQUEIRA, M.F. \& FRANCO, G.A.D.C. 2007. Threats to the cerrado remnants of the state of São Paulo, Brazil. Scientia Agricola 64:355-363.
EMBRAPA. 1982. Levantamento de reconhecimento de media intensidade dos solos e avaliações da aptidão agrícola das terras do Triângulo Mineiro. Boletim de Pesquisa 1. Serviço Nacional de Levantamento e Conservação de Solos. Rio de Janeiro, RJ.

FAHRIG, L. 2003. Effects of habitat fragmentation on biodiversity. Annual Review of Ecology and Systematics 34:487-515.

FISHER, R.A., CORBET, A.S. \& WILLIANS, C.B. 1943. The relation between the number of species and the number of individuals in a random sample of an animal population. Journal of Animal Ecology 12:42-58.

HAY, J.D., BIZERRIL, M.X., CALOURO, A.M., COSTA, E.M.N., FERREIRA, A.A., GASTAL, M.L.A., GOESJUNIOR, C.D., MANZAN, D.J., MARTINS, C.R., MONTEIRO, J.M.G., OLIVEIRA, S.A., RODRIGUES, M.C.M., SEYFFARTH, J.A.S.\& WALTER, B.M.T. 2000. Comparação do padrão da distribuição espacial em escalas diferentes de espécies nativas do cerrado, em Brasília, DF. Revista Brasileira de Botânica 23:341-347.

HOFFMANN, W.A. 2002. Direct and indirect effects of fire on radial growth of cerrado savanna trees. Journal of Tropical Ecology 18:137-142.

HOFFMANN, W.A., ORTHEN, B. \& FRANCO, A.C. 2004. Constraints to seedling success of savanna and forest trees across the savanna-forest boundary. Oecologia 140:252-260.

KLINK, C.A. \& MACHADO, R.B. 2005. Conservation of the Brazilian Cerrado. Conservation Biology 3:707-713.

KÖPPEN, W. 1936. Das geographische System der Klimate. In Handbuch der Klimatologie. (W. Köppen \& R. Geiger, eds.). Berlin, p.1-44.

LAURANCE, W.F. 1999. Ecology and management of fragmented tropical landscapes - introduction and synthesis. Biological Conservation 91:101-107.

LAURANCE, W.F. \& VASCONCELOS, H.L. 2009. Consequências ecológicas da fragmentação florestal na Amazônia. Oecologia Brasiliensis 13:434-451.

LAURANCE, W.F., LOVEJOY, T.E., VASCONCELOS, H.L., BRUNA, E.M., DIDHAM, R.K., STOUFFER, P.C., GASCON, C., BIERREGAARD, R.O., LAURANCE, S.G., \& SAMPAIO, E. 2002. Ecosystem decay of Amazonian forest fragments, a 22-year investigation. Conservation Biology 16:605-618.

LAURANCE, W.F., NASCIMENTO, H.E.M., LAURANCE, S.G., ANDRADE, A., RIBEIRO, J.E.L.S., GIRALDO, J.P., LOVEJOY, T.E., CONDIT, R., CHAVE, J., HARMS, K.E. \& D'ANGELO, S. 2006. Rapid decay of treecommunity composition in Amazonian forest fragments. Proceedings of the National Academy of Sciences of the United States of America 103:19010-19014.

LIMA-RIBEIRO, M.S. 2008. Efeitos de borda sobre a vegetação e estruturação populacional em fragmentos de Cerradão no Sudoeste Goiano, Brasil. Acta Botanica Brasilica 22:535-545. 
MARTINS, C.R., LEITE, L.L. \& HARIDASAN, M. 2004. Capim-gordura (Melinis minutiflora P. Beauv.), uma gramínea exótica que compromete a recuperação de áreas degradadas em unidades de conservação. Revista Árvore 5:739-747.

MCCUNE, B. \& GRACE, J.B. 2002. Analysis of ecological communities. MjM Software Design, Gleneden Beach, Oregon.

MEDEIROS, M.B. \& MIRANDA, H.S. 2005. Mortalidade pós-fogo em espécies lenhosas de campo sujo submetido a três queimadas prescritas anuais. Acta Botanica Brasilica 19:493-500.

MEIRELLES, M.L. \& BARRETO LUIZ, A.J. 1995. Padrões espaciais de árvores de um cerrado em Brasília, DF. Revista Brasileira de Botânica 18:185-189.

MITTERMEIER, R.A., MYERS, N. \& MITTERMEIER, C.G. 1999. Hotspots: earth's biologically richest and most endangered terrestrial ecoregions. Cemex, Mexico City.

MOREIRA, A.G. 2000. Effects of fire protection on savanna structure in Central Brazil. Journal of Biogeography 27:1021-1029.

MYERS, N., MITTERMEIR, R.A., MITTERMEIR, C.G., FONSECA, G.A.B. \& KENTS, J. 2000. Biodiversity hotspots for conservation priorities. Nature 407:853-858.

PIMM, S. \& RAVEN, P. 2000. Extinction by numbers. Nature 403:843-845.

PIVELlO, V.R., CARVALHO, V.M.C., LOPES, P.F., PECCININI, A.A. \& ROSSO, S. 1999. Abundance and distribution of native and alien grasses in a "Cerrado" (Brazilian Savanna) biological reserve. Biotropica 31:71-82.

RATTER, J.A., BRIDGEWATER, S., ATKINSON, R. \& RIBEIRO, J.F. 1996. Analysis of the floristic composition of the Brazilian cerrado vegetation II: Comparison of the woody vegetation of 98 areas. Edinburgh Journal of Botanic 53:153-180.
RATTER, J.A., RIBEIRO, J.F. \& BRIDGEWATER, S. 1997. The Brazilian cerrado vegetation and threats to its biodiversity. Annals of Botany 80:223-230.

RATTER, J.A., BRIDGEWATER, S. \& RIBEIRO, J.F. 2003. Analysis of the floristic composition of the Brazilian cerrado vegetation. III: comparison of the woody vegetation of 376 areas. Edinburgh Journal of Botany 60:57-109.

RIBEIRO, J.R. \& WALTER, B.M.T. 2008. Fitofisionomias do Bioma Cerrado. In Cerrado: ecologia e flora. (S.M. Sano \& S.P. Almeida, eds.). Embrapa-CPAC, Planaltina, p.151-199.

SIEGEL, S. 1956. Nonparametric statistics for the behavioral sciences. McGraw-Hill, New York.

SILVA, M.I.S., GUIMARÃES, E.C. \& TAVARES, M. 2008. Previsão da temperatura média mensal de Uberlândia, MG, com modelos de séries temporais. Revista Brasileira de Engenharia Agrícola e Ambiental 12:480-485.

SPSS. 2000. Systat version 10. SPSS Inc., San Francisco.

TABARELLI, M., MANTOVANI, W. \& PERES, C.A. 1999. Effects of habitat fragmentation on plant guild structure in the montane Atlantic forest of southeastern Brazil. Biological Conservation 91:119-127.

TAVARES, C.E.C. 2005. Fatores críticos à competitividade da soja no Paraná e no Mato Grosso. http://www.conab. gov.br/download/cas/especiais/trabalho $\% 20$ sobre $\% 20$ c Competitividade\%20Soja\%20MT\%20e\%20PR.pdf (acesso em 07/04/2010).

TAYLOR, L.R., KEMPTON, R.A. \& WOIWOD, I.P. 1976. Diversity statistics and the log-series model. Journal of Animal Ecology 45:255-272.

TERBORGH, J. 1992. Maintenance of diversity in tropical forests. Biotropica 24:283-292.

VIEIRA, D.L.M., SCARIOT, A. \& HOLL, K.D. 2006. Effects of habitat, cattle grazing and selective logging on seedling survival and growth in dry forests of central Brazil. Biotropica 39:269-274. 